\title{
Prediction of a quantum anomalous Hall state in Co-decorated silicene
}

\author{
T. P. Kaloni, N. Singh, and U. Schwingenschlögl* \\ Physical Science and Engineering Division, KAUST, Thuwal 23955-6900, Kingdom of Saudi Arabia
}

(Received 3 July 2013; revised manuscript received 23 September 2013; published 9 January 2014)

\begin{abstract}
Based on first-principles calculations, we demonstrate that Co-decorated silicene can host a quantum anomalous Hall state. The exchange field induced by the Co atoms combined with the strong spin-orbit coupling of the silicene opens a nontrivial band gap at the $K$ point. As compared to other transition metals, Co-decorated silicene is unique in this respect, since usually hybridization and spin-polarization induced in the silicene suppress a quantum anomalous Hall state.
\end{abstract}

DOI: 10.1103/PhysRevB.89.035409

PACS number(s): 73.22.Gk, 75.50.Pp, 73.43.Cd

\section{INTRODUCTION}

Silicene is a single layer of $\mathrm{Si}$ atoms arranged in a two-dimensional honeycomb lattice [1] and therefore closely related to graphene. It nowadays attracts considerable attention due to its exotic electronic structure and promising applications in Si nanoelectronics. The chemical similarity between silicene and graphene arises from the fact that $\mathrm{Si}$ and $\mathrm{C}$ belong to the same group in the periodic table. However, $\mathrm{Si}$ is subject to $s p^{3}$ hybridization, whereas pure $s p^{2}$ hybridization is energetically favorable in graphene. Silicene has a buckled structure with two sublattices displaced vertically with respect to each other. The synthesis of silicene nanoribbons on the anisotropic $\operatorname{Ag}(110)$ surface has been studied early [2,3], while recently growth on $\mathrm{Ag}(111)$ has been reported [4,5]. A combined experimental and theoretical study by Fleurence and coworkers [6] on the formation of silicene on $\mathrm{ZrB}_{2}$ thin film has indicated that the buckling is influenced by the interaction with the substrate, leading to a direct electronic band gap at the $\Gamma$ point. Simulations based on density functional theory show that the electronic structure of silicene is governed by a Dirac cone, similar to graphene [7]. Around the Fermi energy, the charge carriers behave as massless Dirac fermions in the $\pi / \pi^{*}$ bands, which approach each other at the $K$ point. The electronic structure of silicene in a perpendicular electric field has been addressed in Ref. [8] and the results of Drummond and coworkers [9] suggest that variation of the electric field with respect to the strength of the spin-orbit coupling induces a transition between a topological and a band insulator.

While the quantum Hall effect is based on an external magnetic field, the quantum anomalous Hall (QAH) effect results from a broken time-reversal symmetry due to an internal magnetization, which can be induced by magnetic doping or adatoms, combined with strong spin-orbit coupling. In topological insulators the quantum spin Hall effect has been observed experimentally [10] and the QAH effect has been predicted [11]. A QAH state also has been predicted by $\mathrm{Yu}$ and coworkers [12] for magnetically doped thin films of topological insulators and by Ezawa for silicene nanoribbons [13]. However, the effect has not been demonstrated for silicene so far, although transition metal adsorption has been studied theoretically in Ref. [14], however, without inclusion of the spin-orbit coupling and on-site Coulomb interaction.

\footnotetext{
*udo.schwingenschlog1@kaust.edu.sa
}

Therefore the QAH state could not be detected in this work. In our present study, we analyze the structural, magnetic, and electronic properties of silicene decorated by the transition metals $\mathrm{Ti}, \mathrm{V}, \mathrm{Cr}, \mathrm{Mn}, \mathrm{Fe}, \mathrm{Co}, \mathrm{Ni}$, or $\mathrm{Cu}$ in comparison to each other. Co decoration is found to result in a unique nature, because all other elements under investigation turn out to inhibit creation of a QAH state for different reasons.

\section{COMPUTATIONAL DETAILS}

We perform geometry optimizations within the generalized gradient approximation including the van der Waals interaction $[15,16]$ as implemented in the Quantum-ESPRESSO package [17]. Moreover, we use a plane-wave cutoff energy of $544 \mathrm{eV}$ and a Monkhorst-Pack $16 \times 16 \times 1 k$ mesh for $4 \times 4 \times 1$ supercells of silicene with a lattice constant of $a=15.44 \AA$ and a vacuum layer of $20 \AA$. Our supercells contain $32 \mathrm{Si}$ atoms and one transition metal atom, such that the density of the impurities is low enough to neglect their mutual interaction. The atomic positions are optimized until all forces have converged to less than $0.003 \mathrm{eV} / \AA$. We calculate the electronic band structures of transition metal decorated silicene by the full potential augmented plane-wave method implemented in the WIEN2K code [18]. The application of a finite on-site Coulomb interaction $U$ is necessary for correctly describing the $d$ electrons of the transition metal atoms, as previous studies suggest that the adsorption geometries and electronic configurations are sensitive to correlation effects $[19,20]$. A value of $U=4 \mathrm{eV}$ is expected to give appropriate results for the transition metal atoms under consideration [21,22] and, therefore, is employed in our calculations. We have also tested different values of the onsite interaction from 3 to $5 \mathrm{eV}$ without finding any influence on our conclusions. The convergence threshold is set to $10^{-4} \mathrm{eV}$ with $R_{\mathrm{mt}} K_{\max }=7$, where $K_{\max }$ is the plane-wave cutoff and $R_{\mathrm{mt}}$ is the smallest muffin-tin radius. A $k$ mesh with 36 points in the irreducible Brillouin zone is used. Silicene has been demonstrated to exist on various substrates. While we do not take into account a specific substrate in our calculations, our results are valid not only for a suspended sample but also in the case that the interaction with the substrate is small.

\section{STRUCTURAL CONSIDERATIONS}

Because of the hexagonal symmetry of silicene, the possible decoration sites for a single atom can be categorized as top, 

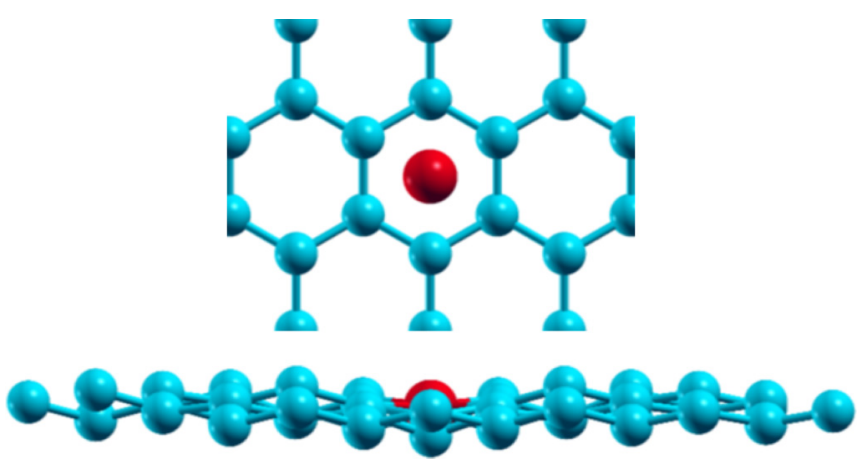

FIG. 1. (Color online) Top and side views of the optimized structure for transition metal decoration of silicene at the hollow site.

bridge, and hollow. While all three choices have been studied, decoration at the bridge site will no longer be considered in the following, because it turns out to be an unstable configuration. The structural optimization demonstrates that a transition metal atom added at the top site shifts close to an original $\mathrm{Si}$ position and displaces the $\mathrm{Si}$ atom. Therefore one metal-Si bond length of $2.43 \AA$ (Ti), $2.41 \AA(\mathrm{V}), 2.38 \AA(\mathrm{Cr}), 2.35 \AA$ $(\mathrm{Mn}), 2.33 \AA(\mathrm{Fe}), 2.30 \AA(\mathrm{Co}), 2.28 \AA(\mathrm{Ni})$, or $2.26 \AA(\mathrm{Cu})$ is realized. In addition, the metal atom is bound to three other $\mathrm{Si}$ atoms with shorter bond lengths of $2.39 \AA$ (Ti), $2.37 \AA(\mathrm{V}), 2.35$ $\AA$ ( $\mathrm{Cr}), 2.31 \AA(\mathrm{Mn}), 2.29 \AA(\mathrm{Fe}), 2.28 \AA(\mathrm{Co}), 2.26 \AA(\mathrm{Ni})$, or $2.24 \AA(\mathrm{Cu})$. The Si-Si bond lengths around the impurity have values of 2.27 to $2.33 \AA$ and thus are slightly modified as compared to pristine silicene (bond length $2.27 \AA$ [23]). The buckling in the silicene layer amounts to 0.34 to $0.52 \AA$ and the binding energy to $E_{b}=3.58 \mathrm{eV}(\mathrm{Ti}), 3.16 \mathrm{eV}(\mathrm{V}), 2.00 \mathrm{eV}$ (Cr), $2.39 \mathrm{eV}(\mathrm{Mn}), 2.91 \mathrm{eV}(\mathrm{Fe}), 3.42 \mathrm{eV}(\mathrm{Co}), 3.05 \mathrm{eV}(\mathrm{Ni})$, or $2.10 \mathrm{eV}(\mathrm{Cu})$. Defining $h$ as the height of the transition metal atom above the silicene plane, we obtain $h=1.90 \AA$ (Ti), 1.80 $\AA(\mathrm{V}), 1.70 \AA(\mathrm{Cr}), 1.20 \AA(\mathrm{Mn}), 1.11 \AA(\mathrm{Fe}), 1.01 \AA(\mathrm{Co})$, $0.91 \AA(\mathrm{Ni})$, or $0.82 \AA(\mathrm{Cu})$. Moreover, the angle $\theta$ between the $\mathrm{Si}$-Si bonds and the normal of the silicene sheet is in pristine silicene $\theta=116^{\circ}$ due to the mentioned mixture of $s p^{2}$ and $s p^{3}$ hybridizations, while around the impurity a wide range of angles, $113^{\circ}$ to $118^{\circ}$, is realized. For decoration at the hollow site, the transition metal atom does not displace a specific $\mathrm{Si}$ atom but stays rather in the center of the Si hexagon, see Fig. 1. It is bound to the six neighboring $\mathrm{Si}$ atoms with bond lengths of 2.26 to $2.35 \AA$. The obtained values of $h$ are listed in Table I. The buckling, the Si-Si bond length, and the angle $\theta$ are found to be slightly modified as compared to decoration at the top site.

The energy difference between the configurations with the transition metal atom at the top and hollow sites is $0.02 \mathrm{eV}$ (Ti), $0.36 \mathrm{eV}(\mathrm{V}), 0.33 \mathrm{eV}(\mathrm{Cr}), 0.01 \mathrm{eV}(\mathrm{Mn}), 0.54 \mathrm{eV}(\mathrm{Fe})$, $0.57 \mathrm{eV}(\mathrm{Co}), 0.52 \mathrm{eV}(\mathrm{Ni})$, and $0.40 \mathrm{eV}(\mathrm{Cu})$, indicating that decoration at the hollow site is always energetically favorable. These values are in reasonable agreement with previous results on transition metal decorated silicene [14], which also applies to the optimized structures. Only the heights $h$ are significantly different, which we attribute to the inclusion of the van der Waal interaction in our structural optimizations and the spinorbit coupling in our electronic structure calculations. It thus is to be expected that our results are more reliable than the previously reported values.

\section{ELECTRONIC STRUCTURE}

In the analysis of the electronic structure, we consider only the energetically favorable hollow site, for the different transition metal impurities. In the case of $\mathrm{Ti}, \mathrm{V}$, and $\mathrm{Cr}$ decoration we obtain total magnetic moments of $2.05 \mu_{B}$, $2.99 \mu_{B}$, and $4.10 \mu_{B}$ per unit cell, respectively, see Table I. It should be noted that the main portion to the magnetic moment comes from the transition metal $d$ orbitals with small contributions form $\mathrm{Si}$ atoms and the interstitial region. We note that the silicene sheet gets significantly polarized, where the magnetic moments of the $\mathrm{Ti}, \mathrm{V}$, and $\mathrm{Cr}$ atoms are aligned ferrimagnetically with respect to the induced Si spins. For half-filling $(\mathrm{Mn})$ and beyond half-filling $(\mathrm{Fe}$ and $\mathrm{Co}$ ), the orientations of the transition metal and Si spins are opposite (antiferromagnetically aligned; negative signs in Table I). In contrast to $\mathrm{Mn}$ and Fe decoration, in the case of Co decoration, the magnetic moment of $0.99 \mu_{B}$ is strongly localized and almost completely due to the Co $d$ orbitals. This fact implies that $\mathrm{Co}$ affects the silicene sheet much less than the other transition metal atoms with larger local magnetic moments. Finally, silicene decorated with $\mathrm{Ni}$ or $\mathrm{Cu}$ turns out to be nonmagnetic, as to be expected.

We consider different coverages of transition metal atoms by employing $1 \times 1 \times 1,2 \times 2 \times 1,3 \times 3 \times 1$, and $4 \times 4 \times 1$ supercells of silicene to which a single transition metal atom

TABLE I. Transition metal decoration at the top site (binding energy) and hollow site (binding energy, structural parameters, total magnetic moment, transition metal $d$ moment, Si moment, interstitial moment, and transition metal occupations for the spin up and down channels).

\begin{tabular}{|c|c|c|c|c|c|c|c|c|c|c|}
\hline & $\begin{array}{c}E_{b} \text { top } \\
(\mathrm{eV})\end{array}$ & $\begin{array}{c}E_{b} \text { bottom } \\
\quad(\mathrm{eV})\end{array}$ & $h(\AA)$ & $\theta\left(^{\circ}\right)$ & $\begin{array}{l}\text { Total } \\
\left(\mu_{B}\right)\end{array}$ & $\begin{array}{l}\text { Metal } d \\
\quad\left(\mu_{B}\right)\end{array}$ & $\operatorname{Si}\left(\mu_{B}\right)$ & $\begin{array}{l}\text { Interstitial } \\
\qquad\left(\mu_{B}\right)\end{array}$ & $\begin{array}{c}\text { Spin up } \\
\text { occupation }\end{array}$ & $\begin{array}{l}\text { Spin down } \\
\text { occupation }\end{array}$ \\
\hline $\mathrm{Ti}$ & 3.58 & 3.60 & 1.40 & $112-118$ & 2.05 & 1.59 & 0.05 & 0.40 & 1.90 & 0.31 \\
\hline V & 3.16 & 3.52 & 1.36 & $113-117$ & 2.99 & 2.55 & 0.02 & 0.41 & 2.80 & 0.25 \\
\hline $\mathrm{Cr}$ & 2.00 & 2.33 & 1.30 & $115-118$ & 4.76 & 4.10 & 0.08 & 0.59 & 4.21 & 0.11 \\
\hline $\mathrm{Mn}$ & 2.39 & 2.40 & 1.10 & $111-115$ & 3.10 & 3.97 & -0.42 & -0.44 & 4.40 & 0.43 \\
\hline $\mathrm{Fe}$ & 2.91 & 3.45 & 0.96 & $111-118$ & 2.10 & 2.69 & -0.25 & -0.33 & 4.39 & 1.70 \\
\hline Co & 3.42 & 3.99 & 0.79 & $113-118$ & 0.99 & 1.16 & -0.07 & -0.10 & 4.48 & 3.32 \\
\hline $\mathrm{Ni}$ & 3.05 & 3.57 & 0.75 & $113-117$ & 0.00 & 0.00 & 0.00 & 0.00 & 4.08 & 4.08 \\
\hline $\mathrm{Cu}$ & 2.10 & 2.50 & 0.66 & $113-117$ & 0.00 & 0.00 & 0.00 & 0.00 & 4.40 & 4.40 \\
\hline
\end{tabular}



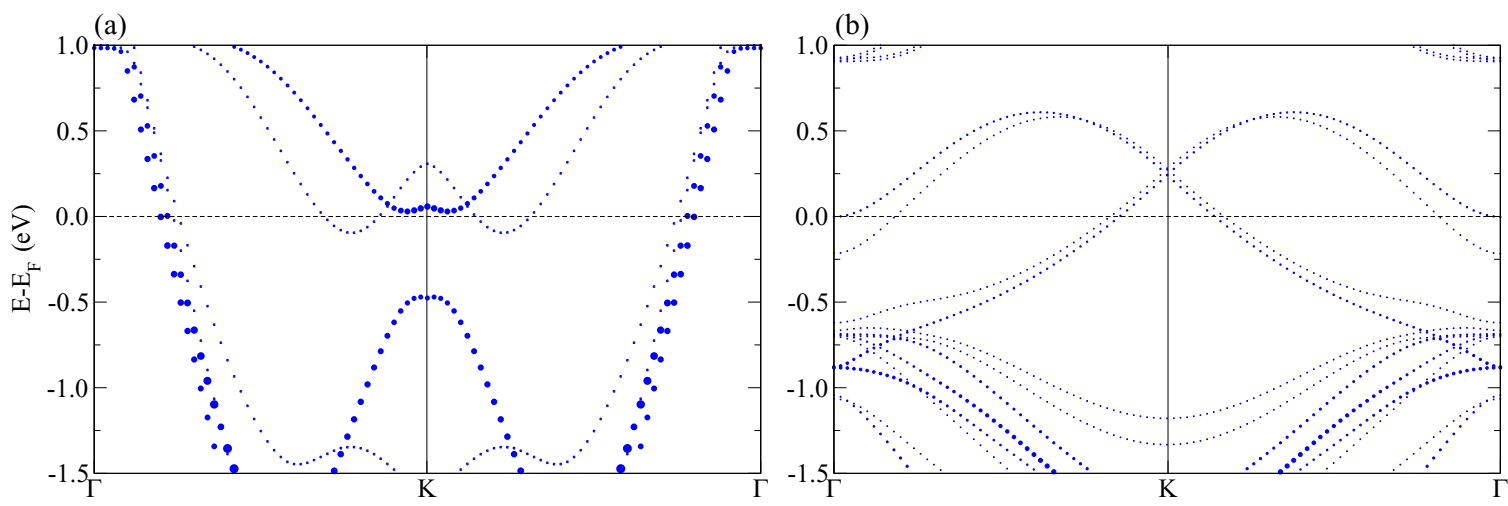

FIG. 2. (Color online) Electronic band structure with weights of the Co $3 d$ states (size of the dots) for decorated silicene (a) $1 \times 1 \times 1$ and (b) $2 \times 2 \times 1$ supercells.

is added. We find in no case besides the $4 \times 4 \times 1$ supercell signs of a QAH state, indicating that interaction between the transition metal atoms counteracts the creation of this state and that, thus, the impurity density has to be sufficiently low. In the case of a high transition metal coverage, see Figs. 2(a) and 2(b) for the example of Co decoration, the Co-Co interaction, coming along with the small separation of only $3.86 \AA$ (periodic boundary conditions), modifies the shape of the band structure in the vicinity of the Fermi energy strongly. For different coverages, accordingly, fundamentally different band structures are obtained. On the other hand, the distance between the transition metal atoms in the $4 \times 4 \times 1$ supercell is large enough to represent the dilute limit. A close similarity of the $4 \times 4 \times 1$ and $5 \times 5 \times 1$ band structures indicates that already in the former case the limit of low impurity density is reached. In the following, we address the $4 \times 4 \times 1$ supercell for this reason.

In the band structures of Ti decorated silicene, see Fig. 3(a), and $\mathrm{V}$ decorated silicene, see Fig. 3(b), a strong hybridization between the transition metal $3 d$ and Si $3 p$ states is observed. Similar findings previously have been reported for $\mathrm{Au}$ and Mo doped graphene $[24,25]$. Due to the hybridization, the QAH effect cannot be realized for Ti and V decoration as the silicene states are perturbed. As a consequence of the relative energetic shift between the impurity and silicene states when another transition metal from the same period of the periodic table is chosen, we can expect that exchange of the impurity can overcome the hybridization problem in the vicinity of the Fermi energy. In the case of $\mathrm{Cr}$ decoration, see Fig. 3(c), we observe the remainder of a Dirac cone with only small transition metal weights, which demonstrates that the states near the Fermi energy are mainly due to the $\mathrm{Si} p_{z}$ orbitals. However, in this case, the local magnetic moment of the impurity is high, see Table I, such that the silicene sheet becomes to some extent spin polarized, which also prevents the creation of a QAH state. The band structure of Mn decorated silicene is addressed in Fig. 3(d). The remainder of a Dirac cone is visible about $0.25 \mathrm{eV}$ below the Fermi energy, reflecting $n$ doping. At the $K$ point, the bands are due to the $\mathrm{Si} p_{z}$ states without hybridization with the $\mathrm{Mn} 3 d$ states. Otherwise, the situation is very similar to the $\mathrm{Cr}$ case, except for the antiferromagnetic polarization of the silicene as mentioned before. In Fig. 3(e), we deal with Fe decorated silicene. The
Dirac cone now is located about $0.12 \mathrm{eV}$ below the Fermi energy at the high symmetry $K$ point, i.e., it is $n$-doped. The $\mathrm{Fe} 3 d$ states likewise do not hybridize with the $\mathrm{Si} 4 p_{z}$ states in the vicinity of the Fermi energy. However, similar to Mn decoration, the high Fe magnetic moment induces significant spin polarization in the silicene, such that no QAH state is realized.

The electronic band structure obtained for Co decorated silicene is shown in Fig. 3(f). We note a tiny energy gap at the $K$ point and the typical band alignment of a $\mathrm{QAH}$ system [12], where the Dirac cone is centered at the Fermi energy. The exchange field due to the Co local magnetic moment breaks the time-reversal symmetry and combined with the strong spin-orbit coupling this results in a QAH state in Co decorated silicene. From the weighted band structure, it is clear that the Dirac cone is essentially purely due to $\mathrm{Si} p_{z}$ orbitals. Therefore the characteristic silicene states are maintained and the way is paved to the QAH state. The magnetic moment of $\mathrm{Co}$ is minimal but finite and therefore just right to break the time-reversal symmetry, while maintaining the electronic structure specific to silicene. Our results clearly demonstrate that a QAH state by transition metal decoration is a rare phenomenon, as in all other cases besides Co the $d-p_{z}$ hybridization and/or the induced spin polarization of the $\mathrm{Si} p_{z}$ electrons perturbs the electronic states. Finally, we mention that for $\mathrm{Ni}$ and $\mathrm{Cu}$ decorated silicene nonmagnetic states are obtained. Interestingly, in both these systems, a Dirac cone is observed below the Fermi level, which could by shifted back by means of a gate voltage. Still, a QAH state could not be induced, since no exchange field is left.

\section{CONCLUSION}

In conclusion, using density functional theory, we find that silicene decoration by the transition metal atoms $\mathrm{Ti}, \mathrm{V}, \mathrm{Cr}$, $\mathrm{Mn}, \mathrm{Fe}, \mathrm{Co}, \mathrm{Ni}$, and $\mathrm{Cu}$ results in occupation of the hollow site of the Si honeycomb. While pristine silicene is subject to spin degeneracy, transition metal decoration can induce substantial spin polarization, which is understood from atomic considerations. We demonstrate that Co decorated silicene is a hybrid material that hosts a QAH state. In the cases of $\mathrm{Ti}$ and $\mathrm{V}$ decoration, a strong hybridization of the transition 

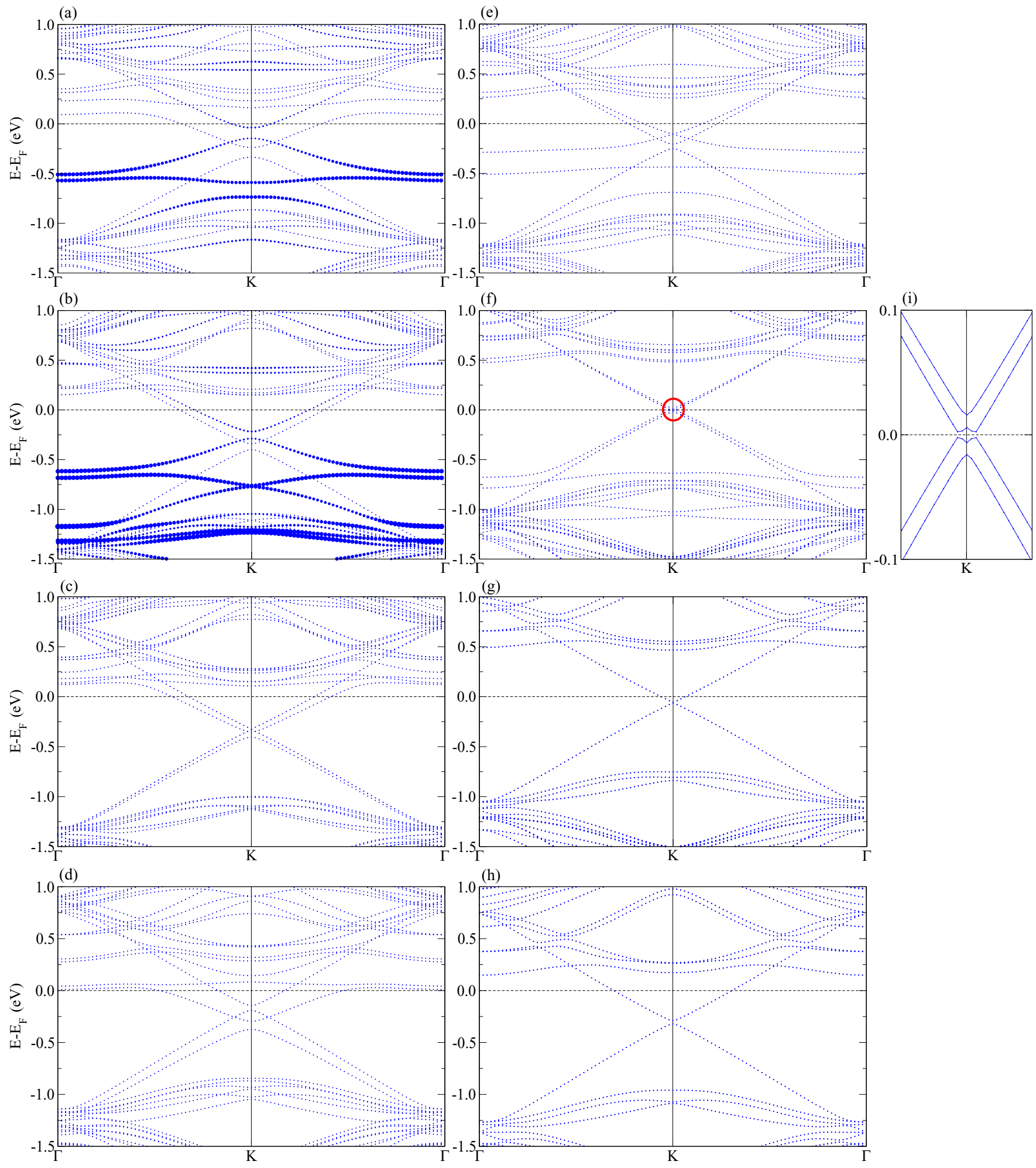

FIG. 3. (Color online) Electronic band structure with weights of the transition metal $3 d$ states (size of the dots) for (a) Ti, (b) V, (c) Cr, (d) $\mathrm{Mn},(\mathrm{e}) \mathrm{Fe}$, (f) $\mathrm{Co},(\mathrm{g}) \mathrm{Ni}$, and (h) $\mathrm{Cu}$ decorated silicene (at the hollow site). (i) Zoom of the marked region of (f).

metal $3 d$ states with the $\mathrm{Si} 3 p_{z}$ states suppresses this state. Because of the large local magnetic moments induced by Mn and $\mathrm{Fe}$, silicene in these cases becomes spin polarized, which also prohibits the formation of the QAH state. $\mathrm{Cr}$ is probably affected by a combination of the two effects, both being weaker but together are enough to perturb the silicene electronic structure sufficiently. $\mathrm{Ni}$ and $\mathrm{Cu}$ decorated silicene are found to be nonmagnetic and therefore also not suitable for our purpose. We have demonstrated that realization of a QAH state is possible in Co decorated silicene, as long as the Co atoms do 
not cluster. On the other hand, both the hybridization and the induced spin polarization typically destroy the characteristic electronic structure of pristine silicene and therefore exclude a QAH state by transition metal decoration. Only in the case of Co decoration, a sufficient but not too large exchange field is achieved that can interact with the strong spin-orbit coupling in silicene. Taking into account effects of the chemical bonding, therefore, results in significant modifications of predictions from a pure model treatment [13].

\section{ACKNOWLEDGMENTS}

We thank M. Tahir for fruitful discussions and KAUST IT for providing computational resources.
[1] G. G. Guzmán-Verri and L. C. LewYanVoon, Phys. Rev. B 76, 075131 (2007).

[2] P. De Padova, C. Quaresima, C. Ottaviani, P. M. Sheverdyaeva, P. Moras, C. Carbone, D. Topwal, B. Olivieri, A. Kara, H. Oughaddou, B. Aufray, and G. Le Lay, Appl. Phys. Lett. 96, 261905 (2010).

[3] P. De Padova, C. Quaresima, P. Perfetti, B. Olivieri, B. Leandri, B. Aufray, S. Vizzini, and G. Le Lay, Nano Lett. 8, 271 (2008).

[4] P. Vogt, P. De Padova, C. Quaresima, J. Avila, E. Frantzeskakis, M. C. Asensio, A. Resta, B. Ealet, and G. Le Lay, Phys. Rev. Lett. 108, 155501 (2012).

[5] B. Feng, Z. Ding, S. Meng, Y. Yao, X. He, P. Cheng, L. Chen, and K. Wu, Nano Lett. 12, 3507 (2012).

[6] A. Fleurence, R. Friedlein, T. Ozaki, H. Kawai, Y. Wang, and Y. Yamada-Takamura, Phys. Rev. Lett. 108, 245501 (2012).

[7] S. Lebegue and O. Eriksson, Phys. Rev. B 79, 115409 (2009).

[8] Z. Ni, Q. Liu, K. Tang, J. Zheng, J. Zhou, R. Qin, Z. Gao, D. Yu, and J. Lu, Nano Lett. 12, 113 (2012).

[9] N. D. Drummond, V. Zolyomi, and V. I. Falko, Phys. Rev B 85, 075423 (2012).

[10] M. König, S. Wiedmann, C. Brüne, A. Roth, H. Buhmann, L. Molenkamp, X. Qi, and S.-C. Zhang, Science 318, 766 (2007).

[11] C. X. Liu, X.-L. Qi, X. Dai, Z. Fang, and S.-C. Zhang, Phys. Rev. Lett. 101, 146802 (2008).

[12] R. Yu, W. Zhang, H.-J. Zhang, S.-C. Zhang, X. Dai, and Z. Fang, Science 329, 61 (2010).

[13] M. Ezawa, Phys. Rev. Lett. 109, 055502 (2012).

[14] X. Lin and J. Ni, Phys. Rev. B 86, 075440 (2012).
[15] S. Grimme, J. Comput. Chem. 27, 1787 (2006).

[16] T. P. Kaloni, Y. C. Cheng, and U. Schwingenschlögl, J. Mater. Chem. 22, 919 (2012).

[17] P. Giannozzi, S. Baroni, N. Bonini, M. Calandra, R. Car, C. Cavazzoni, D. Ceresoli, G. L. Chiarotti, M. Cococcioni, I. Dabo, A. Dal Corso, S. de Gironcoli, S. Fabris, G. Fratesi, R. Gebauer, U. Gerstmann, C. Gougoussis, A. Kokalj, M. Lazzeri, L. Martin-Samos, N. Marzari, F. Mauri, R. Mazzarello, S. Paolini, A. Pasquarello, L. Paulatto, C. Sbraccia, S. Scandolo, G. Sclauzero, A. P. Seitsonen, A. Smogunov, P. Umari, and R. M. Wentzcovitch, J. Phys. Condens. Matt. 21, 395502 (2009).

[18] P. Blaha, K. Schwarz, G. Madsen, D. Kvasicka, and J. Luitz, WIEN2K, An Augmented Plane Wave + Local Orbitals Program for Calculating Crystal Properties (TU Vienna, Vienna, 2001).

[19] T. P. Kaloni, M. Upadhyay Kahaly, and U. Schwingenschlögl, J. Mater. Chem. 21, 18681 (2011).

[20] T. O. Wehling, A. I. Lichtenstein, and M. I. Katsnelson, Phys. Rev. B 84, 235110 (2011).

[21] A. E. Bocquet, T. Mizokawa, T. Saitoh, H. Namatame, and A. Fujimori, Phys. Rev. B 46, 3771 (1992).

[22] P. Wei and Z. Q. Qi, Phys. Rev. B 49, 10864 (1994).

[23] C.-C. Liu, W. Feng, and Y. Yao, Phys. Rev. Lett. 107, 076802 (2011).

[24] D. Marchenko, A. Varykhalov, M. R. Scholz, G. Bihlmayer, E. I. Rashba, A. Rybkin, A. M. Shikin, and O. Rader, Nat. Commun. 3, 1232 (2012).

[25] J. Kang, H.-X. Deng, S.-S. Li, and J. Li, J. Phys.: Condens. Matter 23, 346001 (2011). 